\title{
Filigrane
}

Écoutes psychothérapiques

\section{Transformations intimes. Le nourrisson dans sa famille, Larmor-Plage, de Jeanne Magagna et al. Éditions du Hulblot, 2007. Traduction de Intimate transformations: Babies with Their Families (2005)}

\section{Guy Da Silva}

Volume 18, numéro 1, printemps 2009

Le corps. Sur le divan. Dans le fauteuil I

URI : https://id.erudit.org/iderudit/037726ar

DOI : https://doi.org/10.7202/037726ar

Aller au sommaire du numéro

Éditeur(s)

Revue Santé mentale au Québec

ISSN

1192-1412 (imprimé)

1911-4656 (numérique)

Découvrir la revue

Citer ce compte rendu

Da Silva, G. (2009). Compte rendu de [Transformations intimes. Le nourrisson dans sa famille, Larmor-Plage, de Jeanne Magagna et al. / Éditions du Hublot, 2007. Traduction de Intimate transformations : Babies with Their Families

(2005)]. Filigrane, 18(1), 105-107. https://doi.org/10.7202/037726ar d'utilisation que vous pouvez consulter en ligne. 


\title{
Transformations intimes. Le nourrisson dans sa famille, Larmor-Plage, de Jeanne Magagna et al. ${ }^{1}$
}

\author{
guy da silva
}

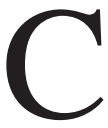

e livre, sous la direction de Jeanne Magagna ${ }^{2}$, est une traduction française parue en 2007 aux Éditions du Hublot (Bretagne) ${ }^{3}$ d'un livre de la collection de la Clinique Tavistock de Londres publié en 2005. Il démontre, à mon avis, de façon plus convaincante que jamais, l'énorme importance pour la suite des choses dans la vie du petit animal humain, de ses toutes premières relations. Cette conviction est devenue pour moi si profonde aujourd'hui, après des années de pratique ${ }^{4}$, que je n'hésite plus maintenant à parler d' « empreintes » pour ces premières expériences émotionnelles ${ }^{5}$.

En effet comment expliquer autrement l'inlassable répétition dans le travail psychanalytique du même mode d'attachement et du même mode de comportement dans la relation transférentielle que celui que l'on peut reconstruire dans la première histoire de la vie du patient adulte. Ce livre, malgré son titre qui paraît s'adresser d'abord aux thérapeutes de famille et aux thérapeutes d'enfants, pourrait tout autant, à mon avis, trouver sa pertinence pour les thérapeutes et analystes d'adultes. Nancy Bakalar d'ailleurs l'exprime très bien dans son chapitre 9 intitulé « Apprendre à partir de l'observation du nourrisson - Comprendre le patient adulte en psychothérapie psychanalytique ».

Dans ce chapitre Nancy Bakalar décrira une de ses patientes de 45 ans dont les angoisses n'ont pu être élucidées que grâce à son étude de bébés hospitalisés dans une unité de soins intensifs pour nouveau-nés. Cette patiente, «Bonnie », était née prématurément et fut placée dans une couveuse à la naissance ; elle était devenue une femme intelligente, diplômée d'université et employée d'une agence immobilière, mais elle continuait de vivre chez ses parents et dans la même chambre, le seul endroit où elle pouvait dormir confortablement !

Ce livre tout entier est ainsi rempli d'anecdotes et de cas cliniques détaillés qui emportent l'adhésion et la conviction sur la pérennité des premières expériences émotionnelles de la toute première enfance.

Le livre est divisé en trois parties : «L'observation du nourrisson au sein de sa famille », «Applications des études en observation du nourrisson », et « Séminaire d'observation du nourrisson ». 


\section{L'observation du nourrisson au sein de sa famille}

Cette première partie décrit, à partir de vignettes détaillées, les observations hebdomadaires de bébés et de jeunes enfants dans leurs familles. Les observations minutieuses de ces relations humaines complexes permettent aux auteurs de lier de façon persuasive ces observations avec la compréhension théorique du développement du jeune enfant. Par exemple : les origines de l'estime de soi dans la petite enfance, la qualité des liens entre frères et sœurs, le rôle de la mère dans le développement de la capacité à tolérer l'émotion, l'expérience qu'a le bébé de luimême et d'autrui, les angoisses œdipiennes et la réaction à la naissance d'un nouveau bébé, ainsi que l'importance de la fonction d'observation encourageant spontanément le parent à observer davantage son bébé ou son jeune enfant, un regard qui se trouve à acquérir une capacité de contenance par identification à la présence de l'observatrice.

\section{Applications des études en observation du nourrisson}

Cette deuxième partie comprend 4 chapitres : contenir l'angoisse dans un service de soins intensifs pour nouveaux-nés ; le rejet comme défense contre la douleur émotionnelle ; l'ombre de votre sourire : intrusion ou engloutissement? et, chapitre très pertinent pour le travail avec les adultes, celui de Nancy Bakalar, sur l'utilisation de l'observation du nourrisson pour « comprendre le patient adulte en psychothérapie psychanalytique ou en psychanalyse ».

\section{Séminaire d'observation du nourrisson}

Quant à la troisième partie, elle porte essentiellement sur le séminaire d'observation du nourrisson et sur l'enseignement de cette observation. Elle démontre l'importance de l'apport du modèle d'affect groupal et comment l'expérience affective du groupe contribue à la compréhension des affects qui ont été éprouvés par le nourrisson au moment où le rapporteur rend compte au groupe de sa rencontre d'observation. Tout a fait fascinant. Encore une fois, on y retient la conviction qu'au-delà des mots rapportés, ce qui importe le plus pour la compréhension, c'est l'expérience émotionnelle éprouvée et transmise qui parle le plus vrai!

guy da silva

830 , pratt, outremont

$\mathrm{qc} \mathrm{h} 2 \mathrm{v} 2 \mathrm{t} 8$

guydasilva@videotron.ca

\section{Notes}

1. Cette recension a d'abord paru dans le Bulletin de la société psychanalytique de Montréal, vol. 21, 1, 2009.

2. Jeanne Magagna est psychanalyste et animatrice d'un séminaire d'observation à la Tavistock de Londres et formée en psychanalyse d'enfants par Esther Bick. Elle a été très influencée à Londres surtout par Herbert Rosenfeld et Donald Meltzer. Elle a été proche des travaux de Henri Rey dont elle a été éditrice de son livre Universaux de la psychanalyse dans le traitement des états psychotiques et borderline. 
Transformations intimes. Le nourrisson dans sa famille, de Jeanne Magagna et al.

3. Éditions du Hublot, 2007. Traduction de Intimate transformations: Babies with Their Families (2005).

4. J'ai déjà eu l'occasion de participer durant une dizaine d'années à un séminaire d'observation de nourrissons. Je souhaite témoigner combien cette expérience a été pour moi révélatrice et a singulièrement modifié la sensibilité affective de mon écoute.

5. Donald Meltzer, Études pour une métapsychologie élargie, Éditions du Hublot, 2006, chapitre 2, «Qu'est-ce qu'une expérience émotionnelle». 